\title{
Fetal alcohol spectrum disorders and their transmission through genetic and epigenetic mechanisms
}

\author{
Edward A. Mead and Dipak K. Sarkar* \\ Rutgers Endocrine Program, Department of Animal Sciences, Rutgers, The State University of New Jersey, New Brunswick, NJ, USA
}

\section{Edited by:}

Feng C. Zhou, Indiana University

School Medicine, USA

Reviewed by:

Ian C. G. Weaver, Dalhousie

University, Canada

Tomas J. Ekstrom, Karolinska

Institutet, Sweden

Laura Sittig, University of Chicago,

USA

\section{${ }^{*}$ Correspondence:}

Dipak K. Sarkar, Rutgers Endocrine Program, Department of Animal Sciences, Rutgers, The State

University of New Jersey, Endocrine Research Building, 67 Poultry Farm Lane, New Brunswick, NJ 08901, USA

e-mail: sarkar@aesop.rutgers.edu
Fetal alcohol spectrum disorders (FASD) are a group of related conditions that arise from prenatal exposure to maternal consumption of the teratogen, ethanol. It has been estimated that roughly $1 \%$ of children in the US suffer from FASD (Sampson etal., 1997), though in some world populations, such as inhabitants of some poorer regions of South Africa, the rate can climb to as high as 20\% (May et al., 2013). FASD are the largest cause of mental retardation in U.S. neonates, and ironically, are entirely preventable. FASD have been linked to major changes in the hypothalamic-pituitary-adrenal (HPA) axis, resulting in lifelong impairments through mental disorders, retardation, and sensitivity to stress. FASD are linked to an impaired immune system which consequently leads to an elevated risk of cancer and other diseases. FASD arise from a complex interplay of genetic and epigenetic factors. Here, we review current literature on the topic to tease apart what is known in these areas particularly emphasizing HPA axis dysfunction and how this ties into new studies of transgenerational inheritance in FASD.

Keywords: fetal alcohol, FASD, HPA axis, proopiomelanocortin, transgenerational epigenetic

\section{INTRODUCTION}

A negative impact from alcohol consumption has been observed since ancient times, leading to cultural prohibitions on alcohol consumption, particularly among women of childbearing years. The ancient Greeks demonstrated some awareness of the risks of alcohol consumption - for example, Plato proposed limiting wine consumption in people under 40 years of age (Abel, 1984). In some ancient societies, such as the Carthaginian Empire, the prohibitions were even written into law (Jones et al., 1973; Abel, 1984). Biblical and Talmudic references suggest that possibly, the ancient Hebrews might have had some awareness that alcohol consumption among fathers and mothers prior to conception could be harmful to the offspring (Abel, 1984). Ancient Vedic writings list prohibitions for drinking by Brahmins (Sharma et al., 2010) suggesting recognition of some negative effects of alcohol, though it is not clear whether an impact on offspring was known. Many sources discussing parental alcohol consumption before modern times focused upon paternal consumption more than maternal (Abel, 1984). With the writings of Francis Bacon in 1627, we see definite concerns about the impact of maternal alcohol consumption on offspring, and in the early 18th century in England, the "gin epidemic" gave rise to commentary that drinking specifically during pregnancy harmed the developing child (Abel, 1984).

Studies in guinea pigs a century ago found that not only did paternal chronic alcohol consumption result in higher mortality rates of offspring, but that the mortality rate was elevated even in the grandchildren of the alcohol-consuming father (Stockard, 1913), giving the first known evidence of a potential multigenerational effect of alcohol exposure. Fetal alcohol syndrome (FAS), the more severe manifestations of the FASD, first began to appear in the medical literature in 1973 (May and Gossage, 2011), and research in the field of fetal alcohol exposure began to take off as the research community launched a coordinated effort into understanding FASD. This led to the recent discovery of a transgenerational impact of fetal alcohol exposure upon stress axis dysfunction. This impact was discovered to be mediated by epigenetic mechanisms carried specifically in the male germline of rats (Govorko et al., 2012).

\section{DYSREGULATION OF THE HPA AXIS IN FETAL ALCOHOL EXPOSED OFFSPRING}

The fetal stage of human life is arguably the most susceptible to harm from alcohol as the fundamental development of organs and pathways occurs in this stage. Not surprisingly, mothers who consume alcohol despite the risks may create severe developmental problems in their offspring. FASD, as its name implies, comprises a wide range of mental, emotional, craniofacial, physiological, and immune disorders which arise from maternal consumption of alcohol. In general, severity of the disorder correlates with the degree of maternal alcohol consumption, though some individuals are more resistant to the effects than others. Individuals with milder forms of FASD may not show any obvious deformities, but may be affected with hyperactivity, depression, anxiety or other disorders that impair quality of life (Schneider et al., 2002). At the other end are individuals with visible deformities, severe retardation, an impaired immune system, impaired metabolic function, and lifelong problems coping with stress, individuals who have FAS (Momino et al., 2012). At the molecular level, many intertwined causal factors contribute to FASD leading to the varied impacts seen among those who suffer. Among the factors, FASD is intimately tied to hyperstress-response and anxiety disorders that are 
connected to the dysregulation of hypothalamic-pituitary-adrenal (HPA) axis functions.

The HPA axis is a complex neuroendocrine loop maintained by crosstalk between the hypothalamus, pituitary, and the peripheral adrenal glands. The paraventricular nucleus (PVN) of the hypothalamus generates corticotrophin-releasing hormone $(\mathrm{CRH})$ and vasopressin, stimulating the production of the precursor polypeptide proopiomelanocortin (POMC) in the anterior lobe of the pituitary. The breakdown of POMC releases adrenocorticotropic hormone (ACTH) initiating the delivery of glucocorticoids (GCs) to peripheral circulation from the adrenal glands. The primary GC produced in the human adrenal cortex is cortisol (corticosterone in rats). Corticosterone can inhibit the production of POMC in the anterior lobe of the pituitary in rats, creating a feedback loop (Eberwine and Roberts, 1984). GCs reduce inflammatory responses through an immunosuppressive action, and stimulate the sympathetic "fight or flight" response giving a rapid, temporary boost to an organism responding to an environmental threat. The autonomic nervous system complements the stress response, either through "fight or flight" (mentioned above), or by "tend and mend," an opposing process through the parasympathetic nervous system. ACTH also stimulates the production of catecholamines (CATs) from the adrenal glands. Epinepherine, also known as adrenaline, and its counterpart, norepinephrine (noradrenaline) are CATs released by the adrenal medulla that activate the sympathetic stress response, leading to many of the common physiological symptoms of stress, such as sweating, dry mouth, and a rapid heartbeat. Though the sympathetic stress response increases survival, it comes at a significant cost in terms of metabolism, the immune system, digestion, and other physiological processes, and cannot be maintained indefinitely (reviewed in Rachdaoui and Sarkar, 2013; Wynne and Sarkar, 2013).

$\beta$-endorphin is another peptide product of POMC generated through the stress response. $\beta$-endorphin is an opioid that can regulate pain, but it also regulates ACTH (another peptide product of POMC; Fratta etal., 1981) and corticotrophin-releasing hormone (CRH; Plotsky, 1986). Hypothalamic $\beta$-endorphin is important to the homeostasis of the stress response. CRH and catecholamines stimulate $\beta$-endorphin release that suppresses the HPA axis response (Boyadjieva et al., 2009). $\delta$ and $\mu$ opioid receptors bind central $\beta$-endorphin, regulating the autonomic nervous system through the PVN. Levels of pituitary $\beta$-endorphin have a smaller role on the autonomic nervous system and are regulated by arginine vasopressin (AVP) and CRH (reviewed by Sarkar et al., 2012).

Proopiomelanocortin neurons in the arcuate nucleus of the hypothalamus play a critical function in regulation of the HPA axis as well as reward pathways and the immune system, through the neuropeptides melanocortin, $\mathrm{ACTH}$, and $\beta$-endorphin derived from the POMC precursor polypeptide (Sarkar et al., 2012). POMC neuronal functions were found to be impaired in fetal alcohol-exposed (FAE) rats (Sarkar et al., 2007; Hellemans et al., 2008; Boyadjieva etal., 2009). Recent experiments showed that replacing $\beta$-endorphin/POMC-producing cells in FAE rats led to an improvement in stress and immune response in the animals, demonstrating a role for POMC in FASD (Boyadjieva et al., 2009).

\section{VARIANT ALLELES OF GENES INVOLVED IN DEVELOPMENT, THE HPA AXIS, AND ALCOHOL METABOLISM PLAY A STRONG ROLE IN FASD SUSCEPTIBILITY AND SYMPTOMS}

Though studies showing heritable damage from fetal alcohol exposure go back at least a century (Stockard, 1913), it was only with the use of molecular biology approaches during the 1980s that science began to unravel the genetic underpinnings of FASD. At the chromosomal level, structural damage was observed at a high frequency among prenatally exposed individuals in a clinical setting. In one study, $8.75 \%(7 / 80)$ of FASD patients in a genetic screening were determined to have chromosomal abnormalities, typically microduplications or microdeletions (Douzgou et al., 2012). Strong evidence for a genetic component to FASD includes a comparison between mono- and dizygotic twins. Monozygotic twins (having the same genome) show 100\% concordance for FASD; that is, if 1 twin has FASD, the other will also have FASD. In comparison, dizygotic twins (having genomes that are moderately different) show only $63 \%$ concordance (Streissguth and Dehaene, 1993), showing that even the modest genetic differences of siblings sharing the same environment lead to a significantly different rate of susceptibility to FASD.

It is known that multiple genetic loci are affected by alcohol, with each variant allele interacting in a complex biological pathway with other genes (Johnson et al., 2006). In FASD, this complexity is multiplied as the genes of the mother can impact the fetal environment and expression of genes of the developing fetus, shaping its susceptibility toward fetal alcohol exposure. Maternal RNA and proteins are present in the oocyte to allow for development to occur in the early embryo until it has developed to the Maternal-to-Zygotic transition (MZT) at which point the embryonic cellular processes take over (Schier, 2007). Maternal hormones have also been shown to play an important role in development of an offspring. These examples show that maternal genes (and their products) can have a strong impact on the development of the fetus beyond contribution of genes alone, dubbed the "maternal effect." By contrast, transmission of a phenotype from the father passed through the father's sperm ("paternal effect") is more limited but has been demonstrated (Fitch et al., 1998). In short, altered parental expression of important genes could result in significant vulnerability or resistance of the offspring to ethanol-induced dysregulation in embryonic development even in the absence of those genes in the child, laying down a dysfunctional or more resistant foundation that lasts a lifetime.

A number of genes have also been identified as potentially playing a direct role in FASD. In a study of rhesus monkeys, it was demonstrated that fetal monkeys carrying a short serotonin transporter gene polymorphic region variation (rh5-HTTLPR), an allele with a functional analog in humans, were particularly susceptible to prenatal alcohol exposure during early gestation, leading to sensory disorders (Schneider et al., 2009). Prior studies indicated that this allele was linked to a greater incidence of irritability and stress-responsiveness in monkey offspring subjected to prenatal alcohol exposure (Kraemer et al., 2008).

Genes coding for alcohol dehydrogenase variants have been found to be particularly relevant for FASD, sometimes resulting in varied incidence or severity of FASD. In some cases, they 
may alter maternal drinking patterns: ADH variants have been linked to alcohol addiction, while other variants may cause mothers to drink less. Alcohol dehydrogenases are found ubiquitously across the kingdoms of living organisms. The products of these genes are involved in converting alcohols $(\mathrm{ROH})$ into aldehydes ( $\mathrm{R}-\mathrm{CHO})$ and ketones (RCOR') through reduction of the coenzyme nicotinamide adenine dinucleotide $\left(\mathrm{NAD}^{+}\right)$into $\mathrm{NADH}$. Humans have six different ADHs, and alcohol dehydrogenase plays an important role in ethanol metabolism and alcohol addiction. Slower metabolizing variants of $\mathrm{ADH} 2$ and -3 have been linked to alcoholism in Asian populations (Osier et al., 1999). ADH1B has a strong link to alcoholism (Li et al., 2011). In FASD, it was discovered that maternal variant alleles of $\mathrm{ADH} 1 \mathrm{~B}, \mathrm{ADH} 1 \mathrm{~B}^{*} 2$, and $\mathrm{ADH}_{1} \mathrm{~B}^{*} 3$ (both relatively common in African-descended populations) which metabolize alcohol rapidly, led to reduced incidence of FASD in offspring, even those without the gene (Warren and Li, 2005; Jacobson et al., 2006). It is known that $\mathrm{ADH}_{1 \mathrm{~B}} 3$ leads to reduced alcohol intake, so possibly it creates an unpleasant association with elevated aldehyde levels through rapid metabolization of alcohol, or the enzyme could help protect offspring by reducing the peak blood alcohol levels, a critical factor in determining the amount of damage done to fetuses (Jacobson etal., 2006), suggesting that these could be underlying mechanisms for reducing the incidence of FASD. In fetal mice exposed to alcohol, variant genotypes of aldh2, an alcohol dehydrogenase gene, have been linked to defects in brain development and vision. This was exacerbated in mice lacking fancd2, which plays a downstream role in processing aldehyde metabolites of alcohol (Langevin et al., 2011). In zebrafish, mars (a gene involved in alcohol metabolism) null fish were linked to developmental face and brain dysfunction during an embryonic alcohol study (McCarthy and Eberhart, 2014). Other genes impacted by ethanol in an embryonic alcohol study conducted in zebrafish include hinfp, plk1, foxd1, and vangl2, which have significance in cellular processes such as translation and the cell cycle, and in early development (McCarthy and Eberhart, 2014). Bioinformatic data mined from the results of published literature searches identified from a screen of over 10,000 candidate genes, a subset of 87 genes within the TGF- $\beta$, MAPK, and Hedgehog signaling pathways which were likely relevant for FASD. These include gnas, and $m s \times 1$, important in apoptosis and cell signaling, fgfr 1-3, important for embryonic bone development, and bmp4, important in myogenesis. Also included were foxg1b, hoxa1, and pax6, important in brain development (Lombard et al., 2007). These genes were examined through pathway, protein-protein and transcription binding analysis and are rich targets for studies into the genetics of FASD. They also fit well with observed phenotypic changes in FASD patients including facial deformities, cardiovascular irregularities, skeletal defects, and brain growth defects (Clarren et al., 1978; Clarren, 1986).

In summary, many genes, either related to alcohol metabolism, or to development and the HPA stress axis, have been identified in relation to FASD. Variants of some of these genes have been shown to contribute to the varied responses seen to fetal alcohol exposure, and it seems likely that other genes also contribute. This may explain in part why the percentage of women who drink during pregnancy is so much higher than the percentage of children who are born with FASD.

\section{FETAL ALCOHOL EXPOSURE CAUSES EPIGENETIC CHANGES CRITICAL TO FASD}

Epigenetics refers to changes in gene expression that do not arise from changes in the underlying DNA sequence. Environmental toxins such as ethanol may impact the expression of genes through altering DNA methylation patterns or modifying histone tails by methylation or acetylation. Epigenetic studies related to FASD are still an emerging field (Haycock, 2009) but have led to the discovery that many symptoms of FASD can be traced back at least in part to aberrant epigenetic marks laid down during gamete production, or during embryonic development under the influence of alcohol. Recent studies have found epigenetic changes due to ethanol that are permanent (Govorko et al., 2012), and they can act broadly across the genome (Kaminen-Ahola et al., 2010). Both DNA methylation and histone modifications, two of the most commonly studied epigenetic mechanisms, can alter the accessibility of DNA to the molecular transcriptional machinery, providing a powerful method for ethanol to create developmental havoc through changing the expression of genes (Renthal and Nestler, 2009).

\section{DNA METHYLATION}

The process of DNA methylation involves the transfer of a methyl group by a DNA methyltransferase (DNMT), utilizing S-adenosyl methionine (SAM), to the $C^{5}$ carbon of a cytosine residue, typically in regions containing strings of $\mathrm{CpG}$ dinucleotides (CpG islands; Bestor, 2000). CpG islands (CGIs) are traditionally defined by having an extended stretch of nucleotides ( $>200$ bases), a $\mathrm{C} / \mathrm{G}$ nucleotide composition above $50 \%$, and an observed $\mathrm{CpG}$ dinucleotide content of $65+\%$ (Gardiner-Garden and Frommer, 1987). Roughly $70 \%$ of annotated promoters show the presence of CGIs that either contain transcription start sites (TSS) or are near them (Saxonov et al., 2006). In vertebrates, CpGs are in low overall abundance and often methylated, however, CpGs in CGIs are frequently unmethylated (Deaton and Bird, 2011). Approximately half of CGIs in mice and humans are associated with TSS (Illingworth et al., 2010; Deaton and Bird, 2011). The remaining, dubbed "orphan CGIs" are themselves often associated with novel promoters (Illingworth et al., 2010; Maunakea et al., 2010).

Methylation of $\mathrm{CpG}$ islands is often regulated during development to control gene expression and the level of methylation correlates with the magnitude of gene inactivation (Doerfler, 1983).

Alcohol may affect DNA methylation through an impact on key methylating enzymes such as DNA methyltransferases (DNMTs). Studies have shown that a downstream metabolite of alcohol breakdown, acetaldehyde, can inhibit DNMT1 (Garro et al., 1991). DNA methylation changes can also occur by altering the interconnected choline, methionine and 5-methyltetrahydrofolate (folate) pathways, which provide key substrates for methylation (Hamid et al., 2009). Alcohol exposure may act through folate (Hidiroglou et al., 1994) and S-adenosylmethionine (SAM; Barak et al., 1993; Lu et al., 2000). Alcohol reduces the absorption of folate from the diet, a vitamin that is necessary for carbon-transfer reactions in 
the methionine-homocysteine pathway, putting limits on DNA methylation (Halsted et al., 2002). Folate deficiency is linked to global DNA hypomethylation (Kim et al., 1997). Choline supplementation reduces FASD symptoms by providing sufficient methyl groups to the methinone-homocysteine pathway (Thomas and Tran, 2012; Bekdash et al., 2013; Wozniak et al., 2013).

Alcohol-mediated aberrant methylation has been documented in at least several developmental genes in alcohol-abusing humans (Bönsch et al., 2006; Hillemacher et al., 2009). In addition, alcohol can act through demethylation (Ponomarev, 2013). DNA methylation changes have been observed to occur during early development. Liu etal. (2009) found that extensive methylation occurred in developmental genes of alcohol-exposed mouse embryos (Liu et al., 2009). Kaminen-Ahola et al. (2010) found that the $\mathrm{A}^{v y}$ gene in mice was hypermethylated following alcohol exposure in early fetal development. Prenatal alcohol exposure studies in mice demonstrated DNA methylation changes resulting in neurofacial and growth defects analogous to FASD in humans (Liu et al., 2009). Alcohol exposure alters DNA methylation profiles in mouse embryos at early neurulation and alters methylation of imprinted genes, known to play roles in the cell cycle, growth, apoptosis, cancer, and in a large number of genes associated with olfaction. These results indicate that gene specific and global changes in DNA methylation occur in response to alcohol during fetal development.

Exposures to ethanol and other toxicants during critical windows in development can be particularly damaging and can cause epigenetic changes in gene expression of the brain that impact the brain for the life of the organism (Kobor and Weinberg, 2011; Ungerer et al., 2013; Nestler, 2014). Alcohol is highly damaging before and during very early development, specifically at three events. When gametes are being produced or are maturing, they are vulnerable to alcohol abuse (Haycock, 2009) as demonstrated by several studies (Ouko et al., 2009; Govorko et al., 2012). This is likely because DNA is being actively repackaged during this period. Also germ cells persist for extended periods of time in the body, providing a longer window for the actions of repeated exposures to environmental toxins like ethanol. A second highly vulnerable period occurs after fertilization but before implantation, when the embryo is undergoing rapid developmental changes and is preparing for implantation. The third occurs at gastrulation, when the three germ layers are being defined. As a result, histone marks are being laid down to specify cell types (Shi and Wu, 2009), and the developing embryo is at its most vulnerable to alcohol at this stage (Armant and Saunders, 1996; Haycock, 2009).

Rat-based studies have found that POMC expression is affected by fetal alcohol exposure, leading to the loss of $\beta$-endorphin producing neurons and a reduction in POMC expression (Chen et al., 2006; Sarkar et al., 2007; Kuhn and Sarkar, 2008). It has been found that ethanol is able to increase DNA methylation of the promoter region of $\mathrm{POMC}$ in fetal pups, changing the epigenetic markings to downregulate POMC expression (Govorko et al., 2012). This is important as POMC neurons, located in the hypothalamus, are critical for bringing stress homeostasis. The perturbation of the HPA axis is a direct cause of many of the symptoms associated with FASD including deficient stress response, depression, anxiety, and impaired immunity (Sarkar, 1996; Pritchard et al., 2002; Raffin-sanson et al., 2003; Sarkar et al., 2012; Rachdaoui and Sarkar, 2013).

In short, DNA methylation has been found to be a strong factor in the incidence of FASD. Alcohol acts through several routes to affect DNA methylation, and seems to be particularly damaging at specific stages of embryonic development resulting in significant changes in methylation that can last a lifetime.

\section{HISTONE MODIFICATION}

Histone modifications are other major biological processes by which epigenetic modification of gene expression occurs. DNA is wound around histones, affecting its ability to interact with transcriptional machinery. Tight packaging results in less interaction. Covalent modifications to the tails of histones 3 and 4 (H3 and $\mathrm{H} 4$ ) are commonly studied. However, $\mathrm{H} 3$ and $\mathrm{H} 4$ are not the only histones that can be epigenetically modified. For $\mathrm{H} 3$ and H4, heterochromatin, the silenced state, is associated with hypoacetylation (de-acetylated state), and di- or trimethylation of the nineth lysine residue on $\mathrm{H} 3$ ( $\mathrm{H} 3 \mathrm{~K} 9 \mathrm{me} 2$ or $\mathrm{H} 3 \mathrm{~K} 9 \mathrm{me} 3$ ). The open state, euchromatin, is associated with acetylated $\mathrm{H} 3$ and $\mathrm{H} 4$, and di- or tri-methylation of the fourth lysine residue of $\mathrm{H} 3$ ( $\mathrm{H} 3 \mathrm{~K} 4 \mathrm{me} 2$ or H3K4me3; Arney and Fisher, 2004). Thus, histone methylation, depending on the target of methylation, can result in a change in expression in either direction. There can be coordinated regulation between histone modifications and other epigenetic mechanisms, including DNA methylation (Jin et al., 2011).

Histone marks have been linked to alcohol consumption (PalBhadra et al., 2007; Pandey et al., 2008). Alcohol causes histone marks to occur in specific genomic locations (Kim and Shukla, 2006). Usually in alcohol studies this has been H3K4me3 (Histone 3 Lysine 4 residue, trimethylated) and acetylation/deacetylation of H3 and H4 (Ponomarev, 2013). Govorko et al. (2012) found that histone deacetylation occurred in fetal rat pups exposed to ethanol, leading to decreased expression of POMC, which partly explains the subsequent disruption of the HPA axis and FASD symptoms observed. See Figure 1 for a summary of current knowledge.

A number of studies into the impact of ethanol on development have been conducted using cell culture models. Cardiac development was affected by ethanol using cardiac progenitor cells. Under ethanol exposure, it was found that heart development was significantly affected, and $\mathrm{H} 3$ acetylation ensued. The effect was dose dependent, leading to greater gene expression changes in higher ethanol doses (Zhong et al., 2010). In fetal neuronal stem cell neurospheres, ethanol was observed to remove histone methylation marks from promoters (either $\mathrm{H} 3 \mathrm{~K} 4 \mathrm{me} 3$ and $\mathrm{H} 3 \mathrm{~K} 27 \mathrm{me} 3$, or both) across 20 candidate genes playing roles in processes such as the regulation of neural stem cell biology, and neural patterning (Veazey et al., 2013). Ethanol also impacted the ES-like bivalent signature of the cells (Veazey et al., 2013). Interestingly, for most genes transcriptional control was not changed by the change in histone modifications (Veazey et al., 2013).

By itself, studies of $\mathrm{H} 3 \mathrm{~K} 4 \mathrm{me} 3$ methylation in relation to alcohol exposure have been found to not be a good predictor of expression (Ponomarev, 2013). Some recent findings have suggested that global chromatin modifications in relation to drug exposure tend 


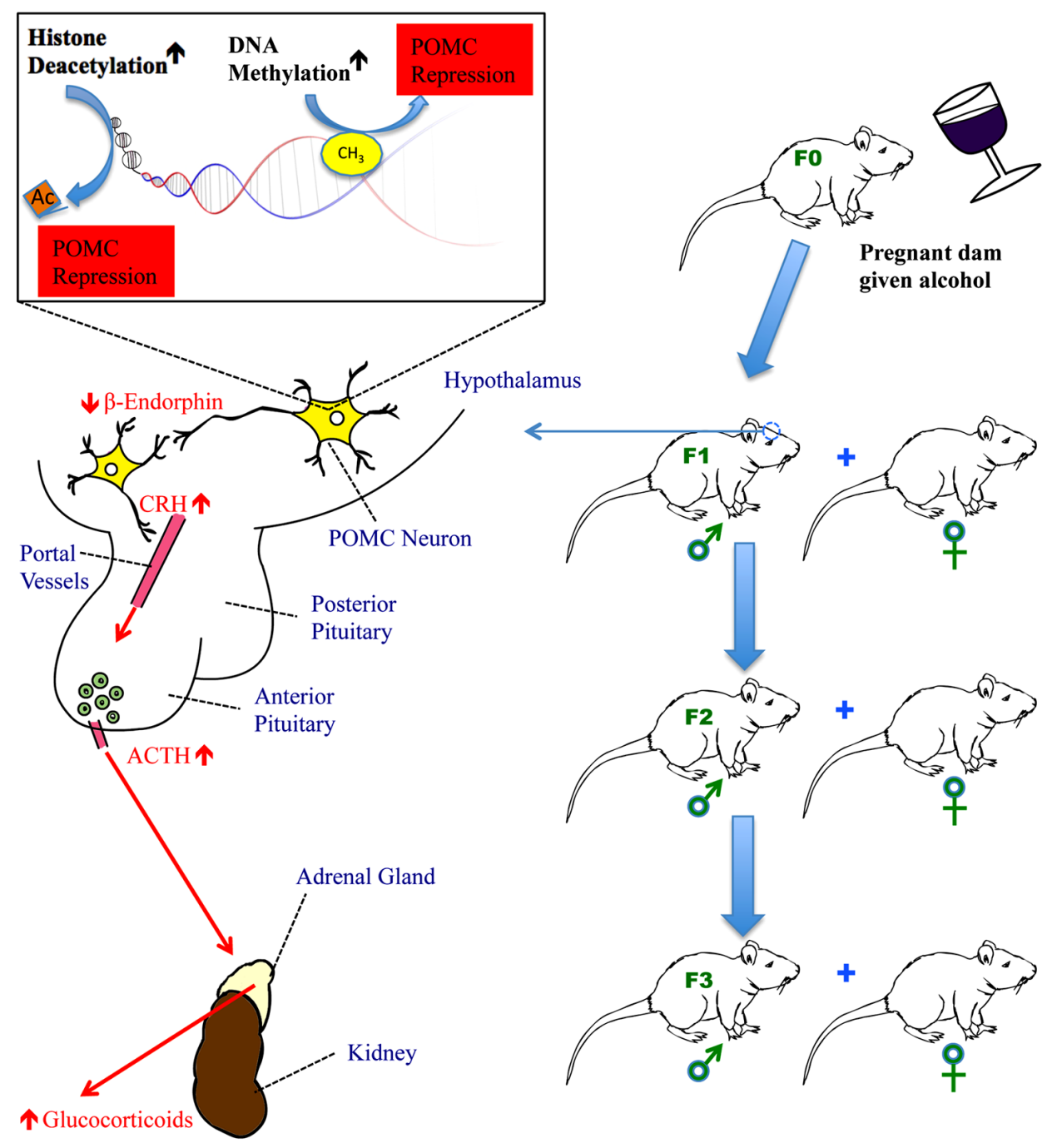

FIGURE 1 |The impact of fetal alcohol exposure on the hypothalamic-pituitary-adrenal (HPA) axis. Fetal alcohol exposure increases DNA methyltransferase activity to methylate $\mathrm{CpGs}$ in the promoter of the POMC gene in the hypothalamus and activates histone deacetylases to remove acetyl groups from histones in the proximity of the POMC gene. Both methylation and deacetylation lead to the repression of $\mathrm{POMC}$ gene transcription. This results in reduced production of $\beta$-endorphin, a peptide derived from POMC gene, in the hypothalamus. Reduced $\beta$-endorphin production disrupts the normal to be transient, and revert back to "normal" states within hours to days after the toxicant is removed suggesting that global changes may not be informative (Nestler, 2014). Though transient in some studies, if damage from aberrant methylation occurs at critical windows such as during fetal development when fundamental structures are being laid down, then at least some of the effects could be permanent.

Epigenetic modifications such as those described above have been linked to many diseases, including major mental disorders such as depression, schizophrenia, bipolar disorder ("manicdepression"), and addiction (Jaenisch and Bird, 2003; Eger et al., 2004; Hsieh and Gage, 2005; Sharma et al., 2008; Maze and Nestler, feedback regulation of the HPA axis causing hyper-response of $\mathrm{CRH}$ following stress and subsequently, increased secretion of $\mathrm{ACTH}$ from the anterior pituitary and glucocorticoids (corticosterone in rats and cortisol in humans) from the adrenal gland. In fetal alcohol-exposed rats, the HPA axis is dysregulated resulting in hyperstress-response, mental dysfunction, and an impaired immune system. POMC gene hypermethylation and its subsequent impact on the HPA axis persist for a minimum of three generations of progeny, carried through the male germline.
2011). Haley et al. (2006) found that the HPA stress axis in infants was impacted from fetal alcohol exposure, likely due to epigenetic changes during gastrulation. Given the links between the HPA axis, FASD and mental disorders, understanding the dysregulation of POMC through epigenetic mechanisms could lead to a better understanding of these diseases and new avenues of treatment.

\section{MicroRNAs}

MicroRNAs (miRNAs) are a class of non-coding RNAs. They are roughly 22 nt in length and function primarily by targeting the $3^{\prime}$ untranslated region (UTR) of transcripts, leading to their downregulation. MiRNAs increasingly have been found to be important 
post-transcriptional gene regulators involved in many biological processes widely across animals and most kingdoms (Reviewed in Berezikov, 2011). The effects of microRNAs in fetal alcohol exposure can occur both at the genetic level and at the level of epigenetics. MiRNA expression has been observed to vary between individuals, leading to changes in predisposition to, and severity of, diseases (Chu et al., 2012; Lukiw, 2013). In some cases, single nucleotide polymorphisms (SNPs) in the genes coding for miRNAs impact their expression and function (Sun et al., 2009). These individual differences could lead to significant differences in the consequences to offspring under maternal alcohol consumption. Alternatively, microRNA expression can be modified through epigenetic mechanisms by ethanol (Miranda, 2012), and, as microRNAs interact directly with epigenetic machinery at multiple levels (reviewed in Singh and Campbell, 2013), it seems likely that maternal ethanol consumption could also affect DNA methylation and histone acetylation/deacetylation via microRNAs. As miRNAs have been implicated in FASD models in rodent studies, particularly in neural development (Wang et al., 2009; Miranda, 2012), changes to miRNA expression likely have a significant role in FASD development in the children of women who abuse alcohol.

There is also evidence of miRNA interaction with the HPA stress axis. Recently, it was demonstrated in mice that paternal stress could impact the HPA stress axis, via epigenetic mechanisms acting on DNA in sperm to transmit heritable changes (Rodgers et al., 2013). Among the microRNAs in the list of those impacted by paternal stress which interact with the HPA stress axis, the expression of miR-29c and miR-204 were found to change in expression due to alcohol exposure in adult rat prefrontal cortex (PFC; Tapocik et al., 2013), whereas miR-29c and -30a were found to be altered in fetal mouse brains during exposure to maternal ethanol (Wang et al., 2009), suggesting that these microRNAs may impact HPA axis development during fetal alcohol exposure.

\section{TRANSGENERATIONAL EPIGENETIC INHERITANCE OF FASD SYMPTOMS$$
\text { HERITABLE EPIGENETIC MODIFICATIONS PLAY A ROLE IN MANY }
$$$$
\text { DISEASES }
$$

Transgenerational epigenetic inheritance, changes carried through the germline (Guerrero-Bosagna et al., 2010; Thornburg et al., 2010), provides an explanation for questions that molecular biologists have not been able to answer through genetics alone. Heritable epigenetic modifications play a key role in some human diseases. Several syndromes in the literature include Angelman, Prader-Willi, and Beckwith-Wiedemann syndrome. These are linked to heritable changes, such as deletions, in specific chromosome regions that undergo genomic imprinting (Adams, 2008). In imprinting, parental differences in epigenetic modifications of specific genes occur in sperm and ova, resulting in parent-specific gene expression. When the contribution to a gene from one parent is silenced through epigenetic mechanisms, deleting the copy from the other parent results in loss of the gene function.

\section{TRANSGENERATIONAL EPIGENETIC MODIFICATIONS ARE RARE}

Transgenerational epigenetic marks on genes are less common than transient marks as most epigenetic signatures are typically lost during gametogenesis. In some cases, certain marks may be retained due to a bias in removal (Morison and Reeve, 1998). Some genes continue to show parental methylation of promoter regions (Borgel et al., 2010). Many epigenetic marks are removed at meiosis (Bond and Finnegan, 2007), and the DNA is methylated again by during early development, often to mark cell type specificity (Shi and Wu, 2009), as there are over 200 different cell types in the body (Alberts et al., 2002).

Prior to fertilization, the male gamete carries the father's germline epigenetic signature. However, when fertilization occurs, most of the male's epigenetic marks are lost. Late in the production of sperm, protamines condense the paternal DNA, protecting it and replacing most histones. However, not all histones are replaced; some histones that remain will keep their prior epigenetic marks (Hammoud et al., 2009; Brykczynska et al., 2010). During the interaction of sperm and egg, after the nuclear membranes fuse and the sperm DNA is deposited, the protamines are lost, replaced with histones from the egg. The DNA from the male enters a more open state- most of the replacing histones are acetylated and the much of the methylation present in the DNA is removed (Oswald et al., 2000; Fulka et al., 2004). Interestingly, some regions seem to be protected from demethylation including imprinted genes (Li, 2002). Following fusion, maternal enzymes re-methylate significant regions of the sperm DNA.

Transgenerational epigenetic effects due to exposure to hazardous chemicals have been documented for 4 or more generations (Anway et al., 2005). To be considered a transgenerational epigenetic effect, the effect must persist for multiple generations. The number of generations required to demonstrate a transgenerational epigenetic effect differs, depending on whether the effect is maternally or paternally transmitted. For maternal transmission, three affected generations of offspring are required to demonstrate a transgenerational epigenetic effect. This is because the fetus (F1) inside the mother (F0) is developing gonads (which give rise to the F2 generation), so a purely environmental effect could in theory directly impact the grandchild through alterations to the gonads of F1, leading to a multigenerational effect, but not demonstrating heritable epigenetic transmission. Therefore, to demonstrate persistence of the effect, three generations (F3) of progeny must be impacted. For paternal transmission, the minimum number of impacted generations is two; that is the grandchild of the founder. Sperm could be directly impacted by an environmental agent, leading to an impact on the F1 offspring, but it would require an epigenetic mechanism to then transmit this effect to the F2 generation (Jirtle and Skinner, 2007).

\section{TRANSGENERATIONAL EPIGENETIC MODIFICATIONS CAUSED BY MATERNAL ALCOHOL CONSUMPTION CAN PROCEED THROUGH THE MALE GERMLINE}

There are a number of multigenerational demonstrations of the heritability of alcohol-related disorders in the literature. In a recent study of Native American women who abused alcohol, F2 generation offspring (that is the grandchildren) of an alcoholabusing woman have a higher tendency to show FAS than those who do F2 progeny of control women (Kvigne et al., 2008). Recently, Govorko etal. (2012) showed that following in utero 
exposure to ethanol, regulatory regions of POMC in the hypothalamus of rats undergo epigenetic modifications: altered histone marks and DNA methylation of the proximal promoter. In addition, histone modifying HDACs and DNA methyltransferases (DNMTs) were shown to be impacted, suggesting a causal relationship between alcohol and epigenetic changes. As a result, POMC neurons are impacted across at least three generations, perturbing the expression of key POMC-derived peptides such as $\beta$-endorphin, and affecting the production of its downstream messenger corticosterone leading to dysregulation of the HPA axis and an elevated response to stress in the adult offspring. This was the first demonstration of a true transgenerational epigenetic effect for prenatal alcohol exposure. Interestingly, Govorko et al. (2012) were able to reverse this effect through HDAC and DNA methylation inhibitors, providing additional support for their conclusion.

There is also evidence that hypomethylation occurs in the sperm of alcoholic men (Ouko et al., 2009). Transmission of the effects of alcohol through the male germline has precedents in the literature for induction of symptoms like those found in FASD. These include mental impairment, cardiac defects, low birth weight, and hyperactivity, compared to controls, as assessed in human epidemiological studies and backed by animal studies (Abel, 2004). This supports the findings of Govorko and colleagues that factors that impact POMC and subsequently affect the HPA axis and FASD, can be transmitted by males through the germline.

\section{SUMMARY AND FUTURE DIRECTIONS}

FASD is caused by a complex interaction of genes and environment, and is regulated by both parental and fetal genes. Some symptoms of FASD are caused by decreased expression of POMC, and it's peptide product, $\beta$-endorphin, important in the HPA stress axis regulation. Recent studies by Govorko et al. (2012) have elucidated that POMC epigenetic changes are transmitted through pups in the male germline descended from fetal alcohol exposed animals for several generations. It is currently unknown how this occurs, as direct changes to the enzymes involved in methylation and deacetylation should also impact female progeny. It is speculated that the non-pairing region of the $\mathrm{Y}$ chromosome, which is euchromatic, may be partly protected from demethylation and could carry the epigenetic modifications to future male progeny.

The reversibility of the POMC system defect that Govorko and colleagues demonstrated via the modulation of the components of the epigenetic machinery may have therapeutic potential. Histone deacetylase inhibitors have proven to be effective in reducing some symptoms of alcohol damage. These act by preventing HDACs from removing acetyl groups from the tails of histones, and ultimately maintain a potentially transcriptionally active state. Rat studies have shown that this can improve the symptoms of FASD (Govorko et al., 2012), and also can function to prevent tolerance and withdrawal in adult rats (Sakharkar et al., 2012).

Choline influences SAM levels, and choline deficiency during development phenotypically mimics folate deficiency (Zeisel, 2004, 2006). Choline chloride supplementation has been successful in reducing the impact of maternal alcohol consumption on developing fetuses (Thomas et al., 2007, 2010; Bekdash et al.,
2013). The use of choline and HDAC or DNMT inhibiting supplements to mitigate FAS symptoms in rats suggests that supplementation could assist at-risk populations during pregnancy, though more studies need to be done in this area. In addition, developments in the understanding of epigenetic regulation of POMC may suggest additional treatment strategies to reduce symptoms of the illness related to the HPA stress axis in adults, and given the links to other disorders, such as cancer and mental disorders, the effects of this research could be far-reaching. The future is promising.

\section{ACKNOWLEDGMENTS}

The authors acknowledge the contribution of Aaron DeLaRosa for helping to draw the cartoon figure. This work is partly supported by National Institute of Health grants R37AA08757 and R01AA016695.

\section{REFERENCES}

Abel, E. (1984). "Is fetal alcohol syndrome a new discovery?" in Fetal Alcohol Syndrome and Fetal Alcohol Effects, ed. E. Abel (New York, NY: Springer Press), 1-28. doi: 10.1007/978-1-4613-2669-4_1

Abel, E. (2004). Paternal contribution to fetal alcohol syndrome. Addict. Biol. 9, 127-133. doi: 10.1080/13556210410001716980

Adams, J. (2008). Imprinting and genetic disease: Angelman, Prader-Willi and Beckwith-Weidemann syndromes. Nat. Educ. 1, 129.

Alberts, B., Johnson, A., Lewis, J., Raff, M., Roberts, K., and Walter, P. (2002). Molecular Biology of the Cell, 4th Edn. New York: Garland Science.

Anway, M. D., Cupp, A. S., Uzumcu, M., and Skinner, M. K. (2005). Epigenetic transgenerational actions of endocrine disruptors and male fertility. Science 308, 1466-1469. doi: 10.1126/science.1108190

Armant, D. R., and Saunders, D. E. (1996). Exposure of embryonic cells to alcohol: contrasting effects during preimplantation and postimplantation development. Semin. Perinatol. 20, 127-139. doi: 10.1016/S0146-0005(96)80080-2

Arney, K. L., and Fisher, A. G. (2004). Epigenetic aspects of differentiation. J. Cell. Sci. 117, 4355-4363. doi: 10.1242/jcs.01390

Barak, A. J., Beckenhauer, H. C., Junnila, M., and Tuma, D. J. (1993). Dietary betaine promotes generation of hepatic S-adenosylmethionine and protects the liver from ethanol-induced fatty infiltration. Alcohol Clin. Exp. Res. 17, 552-555. doi: 10.1111/j.1530-0277.1993.tb00798.x

Bekdash, R. A., Zhang, C., and Sarkar, D. K. (2013). Gestational choline supplementation normalized fetal alcohol-induced alterations in histone modifications, DNA methylation, and proopiomelanocortin (POMC) gene expression in Bendorphin-producing POMC neurons of the hypothalamus. Alcohol Clin. Exp. Res. 37, 1133-1142. doi: 10.1111/acer.12082

Berezikov, E. (2011). Evolution of microRNA diversity and regulation in animals. Nat. Rev. Genet. 12, 846-860. doi: 10.1038/nrg3079

Bestor, T. H. (2000). The DNA methyltransferases of mammals. Hum. Mol. Genet. 9, 2395-2402. doi: 10.1093/hmg/9.16.2395

Bond, D. M., and Finnegan, E. J. (2007). Passing the message on: inheritance of epigenetic traits. Trends Plant Sci. 12, 211-216. doi: 10.1016/j.tplants.2007. 03.010

Bönsch, D., Lenz, B., Fiszer, R., Frieling H., Kornhuber, J., and Bleich, S. (2006). Lowered DNA methyltransferase (DNMT-3b) mRNA expression is associated with genomic DNA hypermethylation in patients with chronic alcoholism. J. Neural Transm. 113, 1299-1304. doi: 10.1007/s00702-005-0413-2

Borgel, J., Guibert, S., Li, Y., Chiba, H., Schübeler, D., Sasaki, H., et al. (2010). Targets and dynamics of promoter DNA methylation during early mouse development. Nat. Genet. 42, 1093-1100. doi: 10.1038/ng.708

Boyadjieva, N. I., Ortigüela, M., Arjona, A., Cheng, X., and Sarkar, D. K. (2009). $\beta$-Endorphin neuronal cell transplant reduces corticotropin releasing hormone hyperresponse to lipopolysaccharide and eliminates natural killer cell functional deficiencies in fetal alcohol exposed rats. Alcohol Clin. Exp. Res. 33, 931-937. doi: 10.1111/j.1530-0277.2009.00911.x

Brykczynska, U., Hisano, M., Erkek, S., Ramos, L., Oakeley, E. J., Roloff, T. C., et al. (2010). Repressive and active histone methylation mark distinct promoters 
in human and mouse spermatozoa. Nat. Struct. Mol. Biol. 17, 679-687. doi: 10.1038/nsmb.1821

Chen, C. P., Kuhn, P., Chaturvedi, K., Boyadjieva, N., and Sarkar, D. K. (2006). Ethanol induces apoptotic death of developing beta-endorphin neurons via suppression of cyclic adenosine monophosphate production and activation of transforming growth factor-betal-linked apoptotic signaling. Mol. Pharm. 69, 706-717. doi: 10.1124/mol.105.017004

Chu, Y. H., Tzeng, S. L., Lin, C. W., Chien, M. H., Chen, M. K., and Yang, S. F. (2012). Impacts of MicroRNA gene polymorphisms on the susceptibility of environmental factors leading to carcinogenesis in oral cancer. PLoS ONE 7:e39777. doi: 10.1371/journal.pone.0039777

Clarren, S. K. (1986). "Neuropathology in fetal alcohol syndrome," in Alcohol and Brain Development, ed. J. R. West (New York: Oxford University Press), 158-166.

Clarren, S. K., Alvord, E. C. Jr., Sumi, S. M., Streissguth, A. P., and Smith, D. W. (1978). Brain malformations related to prenatal exposure to ethanol. J. Pediatr 92, 64-67. doi: 10.1016/S0022-3476(78)80072-9

Deaton, A. M., and Bird, A. (2011). CpG islands and the regulation of transcription. Genes Dev. 25, 1010-1022. doi: 10.1101/gad.2037511

Doerfler, W. (1983). DNA methylation and gene activity. Annu. Rev. Biochem. 52, 93-124. doi: 10.1146/annurev.bi.52.070183.000521

Douzgou, S., Breen, C., Crow, Y. J., Chandler, K., Metcalfe, K., Jones, E., et al. (2012). Diagnosing fetal alcohol syndrome: new insights from newer genetic technologies. Arch. Dis. Child. 97, 812-817. doi: 10.1136/archdischild-2012-302125

Eberwine, J. H., and Roberts, J. L. (1984). Glucocorticoid regulation of proopiomelanocortin gene transcription in the rat pituitary. J. Biol. Chem. 259, 2166-2170.

Eger, G., Liang, G., Aparcio, A., and Jones, P. A. (2004). Epigenetics in human disease and prospects for epigenetic therapy. Nature 429, 457-463. doi: 10.1038/nature02625

Fitch, K. R., Yasuda, G. K., Owens, K. N., and Wakimoto, B. T. (1998). Paternal effects in Drosophila: implications for mechanisms of early development. Curr Top. Dev. Biol. 38, 1-34. doi: 10.1016/S0070-2153(08)60243-4

Fratta, W., Rossetti, Z. L., Poggioli, R., and Gessa, G. L. (1981). Reciprocal antagonism between ACTH1-24 and beta-endorphin in rats. Neurosci. Lett. 24, 71-74. doi: 10.1016/0304-3940(81)90361-X

Fulka, H., Mrazek, M., Tepla, O., and Fulka, J. (2004). DNA methylation pattern in human zygotes and developing embryos. Reproduction 128, 703-708. doi 10.1530/rep.1.00217

Gardiner-Garden, M., and Frommer, M. (1987). CpG islands in vertebrate genomes. J. Mol. Biol. 196, 261-282. doi: 10.1016/0022-2836(87)90689-9

Garro, A. J., McBeth, D. L., Lima, V., and Lieber, C. S. (1991). Ethanol consumption inhibits fetal DNA methylation in mice: implications for the fetal alcohol syndrome. Alcohol Clin. Exp. Res. 15, 395-398. doi: 10.1111/j.15300277.1991.tb00536.x

Govorko, D., Bekdash, R. A., Zhang, C., and Sarkar, D. K. (2012). Male germline transmits fetal alcohol adverse effect on hypothalamic proopiomelanocortin gene across generations. Biol. Psychiatry 72, 378-388. doi: 10.1016/j.biopsych.2012.04.006

Guerrero-Bosagna, C., Settles, M., Lucker, B., and Skinner, M. K. (2010). Epigenetic transgenerational actions of vinclozolin on promoter regions of the sperm epigenome. PLOS ONE 5:e13100. doi: 10.1371/journal.pone. 0013100

Haley, D. W., Handmaker, N. S., and Lowe, J. (2006). Infant stress reactivity and prenatal alcohol exposure. Alcohol Clin. Exp. Res. 30, 2055-2064. doi: 10.1111/j.1530-0277.2006.00251.x

Halsted, C. H., Villanueva, J. A., Devlin, A. M., and Chandler, C. J. (2002). Metabolic interactions of alcohol and folate. J. Nutrit. 132(8 Suppl.), S2367-S2372.

Hamid, A., Wani, N. A., and Kaur, J. (2009). New perspectives on folate transport in relation to alcoholism-induced folate malabsorption-association with epigenome stability and cancer development. FEBS J. 276, 2175-2191. doi: 10.1111/j.1742-4658.2009.06959.x

Hammoud, S. S., Nix, D. A., Zhang, H., Purwar, J., Carrell, D. T., and Cairns, B. R. (2009). Distinctive chromatin in human sperm packages genes for embryo development. Nature 460, 473-478. doi: 10.1038/nature08162

Haycock, P. C. (2009). Fetal alcohol spectrum disorders: the epigenetic perspective. Biol. Reprod. 81, 607-617. doi: 10.1095/biolreprod.108.074690

Hellemans, K. G., Verma, P., Yoon, E., Yu, W., and Weinberg, J. (2008). Prenatal alcohol exposure increases vulnerability to stress and anxiety-like disorders in adulthood. Ann. N. Y. Acad. Sci. 1144, 154-175. doi: 10.1196/annals. 1418.016

Hidiroglou, N., Camilo, M. E., Beckenhauer, H. C., Tuma, D. J., Barak, A. J., Nixon, P. F., et al. (1994). Effect of chronic alcohol ingestion on hepatic folate distribution in the rat. Biochem. Pharmacol. 47, 1561-1566. doi: 10.1016/0006-2952(94) 90532-0

Hillemacher, T., Frieling, H., Hartl, T., Wilhelm, J., Kornhuber, J., and Bleich, S. (2009). Promoter specific methylation of the dopamine transporter gene is altered in alcohol dependence and associated with craving. J. Psychol. Res. 43, 388-392. doi: 10.1016/j.jpsychires.2008.04.006

Hsieh, J., and Gage, F. H. (2005). Chromatin remodeling in neural development and plasticity. Curr. Opin. Cell Biol. 17, 664-671. doi: 10.1016/j.ceb.2005.09.002

Illingworth, R. S., Gruenewald-Schneider, U., Webb, S., Kerr, A. R. W., James, K. D., Turner, D. J., et al. (2010). Orphan CpG islands identify numerous conserved promoters in the mammalian genome. PLoS Genet. 6:e1001134. doi: 10.1371/journal.pgen.1001134

Jacobson, S. W., Carr, L. G., Croxford, J., Sokol, R. J., Li, T. K., and Jacobson, J. L. (2006). Protective effects of the alcohol dehydrogenase-ADH1B allele in children exposed to alcohol during pregnancy. J. Pediatr. 148, 30-37. doi: 10.1016/j.jpeds.2005.08.023

Jaenisch, R., and Bird, A. (2003). Epigenetic regulation of gene expression: how the genome integrates intrinsic and environmental signals. Nat. Genet. 33, 245-254. doi: $10.1038 / \mathrm{ng} 1089$

Jin, B., Li, Y., and Robertson, K. D. (2011). DNA methylation: superior or subordinate in the epigenetic hierarchy? Genes Cancer 2, 607-617. doi: $10.1177 / 1947601910393957$

Jirtle, R., and Skinner, M. K. (2007). Environmental epigenomics and disease susceptibility. Nat. Rev. 8, 253-262. doi: 10.1038/nrg2045

Johnson, C., Drgon, T., Liu, Q. R., Walther, D., Edenberg, H., Rice, J., et al. (2006). Pooled association genome scanning for alcohol dependence using 104,268 SNPs: validation and use to identify alcoholism vulnerability loci in unrelated individuals from the collaborative study on the genetics of alcoholism. Am. J. Med. Genet. B. Neuropsychiatr. Genet. 141B, 844-853. doi: 10.1002/ajmg.b.30346

Jones, K. L., Smith, D. W., Ulleland, C. N., and Streissguth, A. P. (1973). Pattern of malformation in offspring of chronic alcoholic mothers. Lancet 1, 1267-1271. doi: 10.1016/S0140-6736(73)91291-9

Kaminen-Ahola, N., Ahola, A., Maga, M., Mallitt, K. A., Fahey, P., Cox T. C., etal. (2010). Maternal ethanol consumption alters the epigenotype and the phenotype of offspring in a mouse model. PLoS Genet. 6:e1000811. doi: 10.1371/journal.pgen.1000811

Kim, J. S., and Shukla, S. D. (2006). Acute in vivo effect of ethanol (binge drinking) on histone $\mathrm{H} 3$ modifications in rat tissues. Alcohol Alcohol. 41, 126-132. doi: 10.1093/alcalc/agh248

Kim, Y. I., Pogribny, I. P., Basnakian, A. G., Miller, J. W., Selhub, J., James, S. J., et al. (1997). Folate deficiency in rats induces DNA strand breaks and hypomethylation within the p53 tumor suppressor gene. Am. J. Clin. Nutr. 65, 46-52.

Kobor, M. S., and Weinberg, J. (2011). Epigenetics and fetal alcohol spectrum disorders. Alcohol Res. Health 34, 15-37.

Kraemer, G. W., Moore, C. F., Newman, T. K., Barr, C. S., and Schneider, M. L. (2008). Moderate level fetal alcohol exposure and serotonin transporter gene promoter polymorphism affect neonatal temperament and limbic-hypothalamicpituitary-adrenal axis regulation in monkeys. Biol. Psychiatry 63, 317-324. doi: 10.1016/j.biopsych.2007.07.017

Kuhn, P., and Sarkar, D. K. (2008). Ethanol induces apoptotic death of $\beta$-endorphin neurons in the rat hypothalamus by a TGF- $\beta 1$-dependent mechanism. Alcohol Clin. Exp. Res. 32, 706-714. doi: 10.1111/j.1530-0277.2008.00627.x

Kvigne, V. L., Leonardson, G. R., Borzelleca, J., and Welty, T. K. (2008). Characteristics of grandmothers who have grandchildren with fetal alcohol syndrome or incomplete fetal alcohol syndrome. Matern. Child Health J. 12, 760-765. doi: 10.1007/s10995-007-0308-y

Langevin, F., Crossan, G. P., Rosado, I. V., Arends, M. J., and Patel, K. J. (2011). Fancd 2 counteracts the toxic effects of naturally produced aldehydes in mice. Nature 475, 53-58. doi: 10.1038/nature10192

Li, E. (2002). Chromatin modification and epigenetic reprogramming in mammalian development. Nat. Rev. Genet. 3, 662-673. doi: 10.1038/nrg887

Li, H., Gu, S., Han, Y., Xu, Z., Pakstis, A. J., Jin, L., et al. (2011). Diversification of the ADH1B gene during expansion of modern humans. Ann. Hum. Genet. 75, 497-507. doi: 10.1111/j.1469-1809.2011.00651.x 
Liu, Y., Balaraman, Y., Wang, G., Nephew, K. P., and Zhou, F. C. (2009). Alcohol exposure alters DNA methylation profiles in mouse embryos at early neurulation. Epigenetics 4, 500-511. doi: 10.4161/epi.4.7.9925

Lombard, Z., Tiffin, N., Hofmann, O., Bajic, V. B., Hide, W., and Ramsay, M. (2007). Computational selection and prioritization of candidate genes for fetal alcohol syndrome. BMC Genomics 8:389. doi: 10.1186/1471-2164 8-389

Lu, S. C., Huang, Z. Z., Yang, H., Mato, J. M., Avila, M. A., and Tsukamoto, H. (2000). Changes in methionine adenosyltransferase and S-adenosylmethionine homeostasis in alcoholic rat liver. Am. J. Physiol. Gastrointest. Liver Physiol. 279, G178-G185.

Lukiw, W. J. (2013). Variability in micro RNA (miRNA) abundance, speciation and complexity amongst different human populations and potential relevance to Alzheimer's disease (AD). Front. Cell. Neurosci. 7:133. doi: $10.3389 /$ fncel.2013.00133

Maunakea, A. K., Nagarajan, R. P., Bilenky, M., Ballinger, T. J., D’Souza, C. Fouse, S. D., et al. (2010). Conserved role of intragenic DNA methylation in regulating alternative promoters. Nature 466, 253-257. doi: 10.1038/nature 09165

May, P. A., and Gossage, J. P. (2011). Maternal risk factors for fetal alcohol spectrum disorders: not as simple as it might seem. Alcohol Res. Health 34, 15-26.

May, P. A., Blankenship, J., Marais, A. S., Gossage, J. P., Kalberg, W. O., Barnard, R., et al. (2013). Approaching the prevalence of the full spectrum of fetal alcohol spectrum disorders in a South African population-based study. Alcohol Clin. Exp. Res. 37, 818-830. doi: 10.1111/acer.12033

Maze, I., and Nestler, E. J. (2011). The epigenetic landscape of addiction. Ann. N. Y. Acad. Sci. 1216, 99-113. doi: 10.1111/j.1749-6632.2010.05893.x

McCarthy, N., and Eberhart, J. K. (2014). Gene-ethanol interactions underlying fetal alcohol spectrum disorders. Cell Mol. Life Sci. doi: 10.1007/s00018-014-1578-3 [Epub ahead of print].

Miranda, R. C. (2012). MicroRNAs and fetal brain development: implications for ethanol teratology during the second trimester period of neurogenesis. Front Genet. 3:77. doi: 10.3389/fgene.2012.00077

Momino, W., Félix, T. M., Abeche, A. M., Zandoná, D. I., Scheibler, G. G., Chambers C., et al. (2012). Maternal drinking behavior and fetal alcohol spectrum disorders in adolescents with criminal behavior in southern Brazil. Genet. Mol. Biol. 35(Suppl. 4), 960-965. doi: 10.1590/S1415-47572012000600011

Morison, I. M., and Reeve, A. E. (1998). A catalogue of imprinted genes and parentof-origin effects in humans and animals. Hum. Mol. Genet. 7, 1599-1609. doi: 10.1093/hmg/7.10.1599

Nestler, E. (2014). Epigenetic mechanisms of drug addiction. Neuropharmacology 76B, 259-268. doi: 10.1016/j.neuropharm.2013.04.004

Osier, M. V., Pakstis, A. J., Kidd, J. R., Lee, J. F., Yin, S. J., Ko, H. C., et al. (1999). Linkage disequilibrium at the $\mathrm{ADH} 2$ and $\mathrm{ADH} 3$ loci and risk of alcoholism. Am J. Hum. Genet. 64, 1147-1157. doi: 10.1086/302317

Oswald, J., Engemann, S., Lane, N., Mayer, W., Olek, A., Fundele, R., et al. (2000) Active demethylation of the paternal genome in the mouse zygote. Curr. Biol. 10, 475-478. doi: 10.1016/S0960-9822(00)00448-6

Ouko, L. A., Shantikumar, K., Knezovich, J., Haycock, P., Schnugh, D. J., and Ramsay, M. (2009). Effect of alcohol consumption on CpG methylation in the differentially methylated regions of H19 and IG-DMR in male gametes: implications for fetal alcohol spectrum disorders. Alcohol Clin. Exp. Res. 33, 1615-1627. doi: 10.1111/j.1530-0277.2009.00993.x

Pal-Bhadra, M., Bhadra, U., Jackson, D. E., Mamatha, L., Park, P., and Shukla, S. D. (2007). Distinct methylation patterns in histone H3 at Lys-4 and Lys-9 correlate with up- and down-regulation of genes by ethanol in hepatocytes. Life Sci. 81, 979-987. doi: 10.1016/j.lfs.2007.07.030

Pandey, S., Rajesh, U., Zhang, H., Tang, L., and Prakash, A. (2008). Brain chromatin remodeling: a novel mechanism of alcoholism. J. Neurosci. 28, 3729-3737. doi: 10.1523/JNEUROSCI.5731-07.2008

Plotsky, P. M. (1986). Opioid inhibition of immunoreactive corticotropin-releasing factor secretion into the hypophysial-portal circulation of rats. Reg. Peptides 16, 235-242. doi: 10.1016/0167-0115(86)90022-4

Ponomarev, I. (2013). Epigenetic control of gene expression in the alcoholic brain. Alcohol Res. 35, 69-76.

Pritchard, L. E., Turnbull, A. V., and White, A. (2002). Pro-opiomelanocortin processing in the hypothalamus: impact on melanocortin signaling and obesity. J. Endocrinol. 172, 411-421. doi: 10.1677/joe.0.1720411
Rachdaoui, N., and Sarkar, D. K. (2013). Effects of alcohol on the endocrine system. Endocrinol. Metab. Clin. N. Am. 42, 593-615. doi: 10.1016/j.ecl.2013.05.008

Raffin-sanson, M. L., de Keyser, Y., and Bertagna, X. (2003). Proopiomelanocortin, a polypeptide precursor with multiple functions: from physiology to pathological conditions. Euro J. Endocrinol. 149, 79-90. doi: 10.1530/eje.0. 1490079

Renthal, W., and Nestler, E. J. (2009). Chromatin regulation in drug addiction and depression. Dialog Clin. Neurosci. 11, 257-268.

Rodgers, A. B., Morgan, C. P., Bronson, S. L., Revello, S., and Bale, T. L. (2013). Paternal stress exposure alters sperm microRNA content and reprograms offspring HPA stress axis regulation. J. Neurosci. 33, 9003-9012. doi: 10.1523/JNEUROSCI.0914-13.2013

Sakharkar, A. J., Zhang, H., Tang, L., Shi G., and Pandey, S. C. (2012). Histone deacetylases (HDAC)-induced histone modifications in the amygdala: a role in rapid tolerance to the anxiolytic effects of ethanol. Alcohol Clin. Exp. Res. 36, 61-71. doi: 10.1111/j.1530-0277.2011.01581.x

Sampson, P. D., Streissguth, A. P., Bookstein, F. L., Little, R. E., Clarren, S. K., Dehaene, P., et al. (1997). Incidence of fetal alcohol syndrome and prevalence of alcohol-related neurodevelopmental disorder. Teratology 56, 317-326. doi: 10.1002/(SICI)1096-9926(199711)56:5<317::AID-TERA5>3.0.CO;2-U

Sarkar, D. K. (1996). Neuroendocrine - immune axis of alcoholics. Symposium. Alcohol Clin. Exp. Res. 20, 256A-259A.

Sarkar, D. K., Kuhn, P., Marano, J., Chen, C., and Boyadjieva, N. (2007). Alcohol exposure during the developmental period induces $B$-endorphin neuronal death and causes alteration in the opioid control of stress axis function. Endocrinology 148, 2828-2834. doi: 10.1210/en.2006-1606

Sarkar, D. K., Murugan, S., Zhang, C., and Boyadjieva, N. (2012). Regulation of cancer progression by $\beta$-endorphin neuron. Cancer Res. 72, 836-840. doi: 10.1158/0008-5472.CAN-11-3292

Saxonov, S., Berg, P., and Brutlag, D. L. (2006). A genome-wide analysis of CpG dinucleotides in the human genome distinguishes two distinct classes of promoters. Proc. Natl. Acad. Sci. U.S.A. 103, 1412-1417. doi: 10.1073/pnas.0510 310103

Schier, A. F. (2007). The maternal-zygotic transition: death and birth of RNAs Science 316, 406-407. doi: 10.1126/science.1140693

Schneider, M. L., Moore, C. F., Kraemer, G. W., Roberts, A. D., and DeJesus, O. T. (2002). The impact of prenatal stress, fetal alcohol exposure, or both on development: perspectives from a primate model. Psychoneuroendocrinology 27, 285-298. doi: 10.1016/S0306-4530(01)00050-6

Schneider, M. L., Moore, C. F., Larson, J. A., Barr, C. S., DeJesus, O. T., and Roberts, A. D. (2009). Timing of moderate level prenatal alcohol exposure influences gene expression of sensory processing behavior in rhesus monkeys. Front. Integr. Neurosci. 3:30. doi: 10.3389/neuro.07.030.2009

Sharma, R. P., Grayson, D. R., and Gavin, D. P. (2008). Histone deacetylase 1 expression is increased in the prefrontal cortex of schizophrenia subjects: analysis of the National Databank microarray collection. Schizophr. Res. 98, 111-117. doi: 10.1016/j.schres.2007.09.020

Sharma, H. K., Tripathi, B. M., and Pelto, P. J. (2010). The evolution of alcohol use in India. AIDS Behav. 14(Suppl. 1), S8-S17.

Shi, L., and Wu, J. (2009). Epigenetic regulation in mammalian preimplantation embryo development. Reprod. Biol. Endocrinol. 7, 59. doi: 10.1186/14777827-7-59

Singh, P. K., and Campbell, M. J. (2013). The interactions of microRNA and epigenetic modifications in prostate cancer. Cancers (Basel) 5, 998-1019. doi: $10.3390 /$ cancers 5030998

Stockard, C. (1913). The effect on the offspring of intoxicating the male parent and the transmission of the defects to subsequent generations. Am. Nat. 47, 641-682. doi: $10.1086 / 279379$

Streissguth, A. P., and Dehaene, P. (1993). Fetal alcohol syndrome in twins of alcoholic mothers: concordance of diagnosis and IQ. Am. J. Med. Genet. 47, 857-861. doi: 10.1002/ajmg.1320470612

Sun, G., Yan, J., Noltner, K., Feng, J., Li, H., Sarkis, D. A., et al. (2009). SNPs in human miRNA genes affect biogenesis and function. RNA 15, 1640-1651. doi: 10.1261/rna.1560209

Tapocik, J. D., Solomon, M., Flanigan, M., Meinhardt, M., Barbier, E., Schank, J. R., et al. (2013). Coordinated dysregulation of mRNAs and microRNAs in the rat medial prefrontal cortex following a history of alcohol dependence. Pharmacogenomics J. 13, 286-296. doi: 10.1038/tpj.2012.17 
Thomas, J. D., Biane, J. S., O’Bryan, K. A., O’Neill, T. M., and Dominguez, H. D. (2007). Choline supplementation following third-trimester equivalent alcohol exposure attenuates behavioral alterations in rats. Behav. Neurosci. 121, 120-130. doi: 10.1037/0735-7044.121.1.120

Thomas, J. D., Idrus, N. M., Monk, B. R., and Dominquez, H. D. (2010). Prenatal choline supplementation mitigates behavioral alterations associated with prenatal alcohol exposure in rats. Birth Defect. Res. A Clin. Mol. Teratol. 88, 827-837. doi 10.1002/bdra.20713

Thomas, J. D., and Tran, T. D. (2012). Choline supplementation mitigates trace, but not delay, eyeblink conditioning deficits in rats exposed to alcohol during development. Hippocampus 22, 619-630. doi: 10.1002/hipo.20925

Thornburg, K. L., Shannon, J., Thuillier, P., and Turker, M. S. (2010). In utero life and epigenetic predisposition for disease. Adv. Genet. 71, 57-78. doi: 10.1016/B9780-12-380864-6.00003-1

Ungerer, M., Knezovich, J., and Ramsay, M. (2013). In utero alcohol exposure, epigenetic changes, and their consequences. Alcohol Res. 35, 37-46.

Veazey, K. J., Carnahan, M. N., Muller, D., Miranda, R. C., and Golding, M. C. (2013). Alcohol-induced epigenetic alterations to developmentally crucial genes regulating neural stemness and differentiation. Alcohol. Clin. Exp. Res. 37, 11111122. doi: 10.1111/acer.12080

Wang, L. L., Zhang, Z., Li, Q., Yang, R., Pei, X., Xu, Y., et al. (2009). Ethanol exposure induces differential microRNA and target gene expression and teratogenic effects which can be suppressed by folic acid supplementation. Hum. Reprod. 24, 562579. doi: 10.1093/humrep/den439

Warren, K. R., and Li, T. K. (2005). Genetic polymorphisms: impact on the risk of fetal alcohol spectrum disorders. Birth Defects Res. A Clin. Mol. Teratol. 73, 195-203. doi: 10.1002/bdra.20125

Wozniak, J. R., Fuglestad, A. J., Eckerle, J. K., Kroupina, M. G., Miller, N. C., Boys, C. J., et al. (2013). Choline supplementation in children with fetal alcohol spectrum disorders has high feasibility and tolerability. Nutr. Res. 33, 897-904. doi: 10.1016/j.nutres.2013.08.005
Wynne, O., and Sarkar, D. K. (2013). "Stress and neuroendocrine-immune interaction: a therapeutic role for $\beta$-endorphin," in The Wiley-Blackwell Handbook of Psychoneuroimmunology, eds A. W. Kusnecov and H. Anisman (Chichester: John Wiley \& Sons Ltd), 198-211. doi: 10.1002/9781118314 814.ch 10

Zeisel, S. H. (2004). Nutritional importance of choline for brain development. J. Am. Coll. Nutr. 23, 621S-626S. doi: 10.1080/07315724.2004.10719433

Zeisel, S. H. (2006). Choline: critical role during fetal development and dietary requirements in adults. Annu. Rev. Nutr. 26, 229-250. doi: 10.1146/annurev.nutr.26.061505.111156

Zhong, L., Zhu, J., Lv, T., Chen, G., Sun, H., Yang, X., et al. (2010). Ethanol and its metabolites induce histone lysine 9 acetylation and an alteration of the expression of heart development-related genes in cardiac progenitor cells. Cardiovasc. Toxicol. 10, 268-274. doi: 10.1007/s12012-010-9081-z

Conflict of Interest Statement: The authors declare that the research was conducted in the absence of any commercial or financial relationships that could be construed as a potential conflict of interest.

Received: 21 March 2014; accepted: 09 May 2014; published online: 02 June 2014. Citation: Mead EA and Sarkar DK (2014) Fetal alcohol spectrum disorders and their transmission through genetic and epigenetic mechanisms. Front. Genet. 5:154. doi: 10.3389/fgene.2014.00154

This article was submitted to Epigenomics and Epigenetics, a section of the journal Frontiers in Genetics.

Copyright (C) $2014 \mathrm{Mead}$ and Sarkar. This is an open-access article distributed under the terms of the Creative Commons Attribution License (CC BY). The use, distribution or reproduction in other forums is permitted, provided the original author(s) or licensor are credited and that the original publication in this journal is cited, in accordance with accepted academic practice. No use, distribution or reproduction is permitted which does not comply with these terms. 